\title{
PROCEEDINGS OF THE DUTCH SOCIETY OF CLINICAL PHARMACOLOGY AND BIOPHARMACY MEETING OF APRIL 13TH 2005
}

\begin{abstract}
Relationship between serotonergic antidepressants and worsening of symptoms of Parkinson's disease

M. E. L. Arbouw, ${ }^{1,2}$ K. L. L. Movig, ${ }^{1}$ C. Neef, ${ }^{1}$ H.-J. Guchelaar ${ }^{3}$ \&

A. C. G. Egberts ${ }^{2}$

'Department of Clinical Pharmacy, Medisch Spectrum Twente,

Enschede, The Netherlands; ${ }^{2}$ Department of

Pharmacoepidemiology and Pharmacotherapy, Utrecht Institute

for Pharmaceutical Sciences (UIPS), Utrecht, The Netherlands

and ${ }^{3}$ Department of Clinical Pharmacy and Toxicology, Leiden

University Medical Centre, Leiden, The Netherlands
\end{abstract}

Background: Selective reuptake inhibitors (SSRIs) are widely accepted as first choice treatment for depression. However, using SSRIs for depression treatment in patients with Parkinson's disease (PD) has frequently been discussed. A possible association between the use of serotonergic antidepressants and worsening of symptoms of Parkinson's disease has been described.

Objectives: The objective of our study was to determine a relationship between initial use of serotonergic antidepressant and worsening of symptoms of Parkinson's disease.

Methods: A retrospective follow-up study was performed using the PHARMO database. Patients of 40 years or older who started with an antidepressant and who had used levodopa for at least 180 days before the index date were included. Patients initially using antipsychotics, metoclopramide, cinnarizine or flunarizine in the period from 180 days before until 180 days after start of follow-up were excluded. Also patients with only one prescription of an antidepressant were excluded.

Start of follow-up was the first dispensing date of the antidepressant. The time of follow-up was 180 days, until the final dispensing date of the antidepressant or until the final dispensing date of any antiparkinsonian drug, which ever came first. Antidepressants were classified in two ways: in three groups according to chemical class (SSRI, TCA and other antidepressants) and in three groups according to the extent (high, intermediate or low) of inhibition of serotonin reuptake. The main outcome measure was a change of antiparkinsonian drug treatment during follow-up. A change was defined as a change in the daily dosage of any antiparkinsonian drug, or when a new antiparkinsonian drug was started. Hazard ratios were calculated using a Cox's proportional hazard model.

Results: The study population consisted of 94, 104 and 42 patients using antidepressants categorized as SSRI, TCA and other antidepressants respectively and consisted of 69,130 and 41 patients using antidepressants categorized as high, intermediate and low serotonin reuptake inhibitors respectively. The hazard ratio for the main outcome measure comparing SSRI with TCA users was 1.4 (95\% CI 0.9-2.2) and comparing other antidepressant with TCA users was 1.8 (95\% CI 1.1-3.2). The hazard ratio comparing high versus low serotonin reuptake inhibition antidepressants was 0.7 (95\% CI 0.4-1.3) and comparing intermediate versus low serotonin reuptake inhibition antidepressants was 0.8 (95\% CI 0.5-1.4).

Conclusions: Worsening of symptoms of Parkinson's disease among patients using serotonergic antidepressants can not primarily be explained by the extent of serotonin reuptake inhibition.

\section{MDR-1 and CYP 3A gene polymorphisms and 12H AUC pharmacokinetics of Tacrolimus}

R. O. den Buijsch, ${ }^{1}$ O. Bekers, ${ }^{1}$ M. Christiaans, ${ }^{2}$ L. Stolk, ${ }^{3}$

S. Cheung, ${ }^{2}$ M. van Dieijen-Visser, ${ }^{1}$ J. van Hooff ${ }^{2}$ \& J. de Vries ${ }^{1,4}$

Dpts. of Clinical Chemistry', Internal Medicine², Clinical

Pharmacy and Toxicology 3 , and Biochemical and Clinical

Genetics ${ }^{4}$ University Hospital of Maastricht, The Netherlands

Introduction: There exists a large inter- and intrapatient variability in oral pharmacokinetics of Tacrolimus (Tac). Both polymorphisms of the P-glycoprotein system (MDR-1), regulating the intestinal uptake of Tac, and of the Cytochrome P450 enzyme system (CYP3A4, CYP3A5), regulating the elimination of Tac, may be of importance. It has been shown that CYP3A, but not the MDR-1 [C3435T] polymorphism, influences the dose-normalised trough level $\left(\mathrm{dnC}_{0}\right)^{1}$. No information exists about the correlation between these polymorphisms and the AUC, $\mathrm{C}_{\max }$ and $\mathrm{T}_{\max }$.

Methods: Patients: 38 Caucasian renal transplant recipients with a stable tacrolimus dose and trough-level were tested.
Pharmacokinetics: a 12-hour time-concentration profile of Tac was performed. Tac was determined with an immunoassay (MEIA, Abbott).

Genomics: Single nucleotide polymorphisms (SNPs) of MDR-1 [C3435T], CYP3A4 [A-392G] and CYP3A5 [A6986G] were determined using real-time PCR fluorescence resonance energy transfer (FRET) assays on the LightCycler. The influence of the polymorphisms on the dose normalised (dn) AUC, $\mathrm{C}_{\max }, \mathrm{T}_{\max }$ and $\mathrm{dnC}_{0}$ was analysed.

Statistics: Data are given as median values. Between group differences were calculated by use of the Kruskal-Wallis H-test.

Results: The allele distribution of the MDR-1 [C3435T] polymorphism is in agreement with literature, while the frequency of the CYP3A5 6986AG genotype is high. There is no correlation between MDR-1 and CYP3A genes. DnAUC and $\mathrm{C}_{\max }$ of patients with the MDR-1 3435CC genotype were lowest but not significantly. Because of the strong correlation we combined the two CYP3A genotypes. As can be seen in Table 1 both CYP3A4 and 3A5 polymorphisms exhibited significant effect on dnAUC and $\mathrm{dnC}_{0}$. 
Table 1

Overview results. Median values; $\mathrm{P}<$ 0.001

\begin{tabular}{|c|c|c|c|c|c|c|}
\hline $\begin{array}{l}\text { Gene } \\
\text { polymorphism }\end{array}$ & $\begin{array}{l}\mathrm{N} \\
\text { no }\end{array}$ & Alleles & $\begin{array}{l}\text { dnAUC } \\
\text { ng*uur/ } \\
\text { kg*mL }\end{array}$ & $\begin{array}{l}\mathrm{C}_{\max } \\
\mathrm{ng}\end{array}$ & $\begin{array}{l}\mathrm{T}_{\max } \\
\text { uur }\end{array}$ & $\begin{array}{l}\mathrm{dnC}_{0} \\
\mathrm{ng} / \mathrm{kg}\end{array}$ \\
\hline MDR-1 & 9 & $\mathrm{CC}$ & 1333 & 33.0 & 1.0 & 55.2 \\
\hline [C3435T] & $\begin{array}{l}18 \\
11\end{array}$ & $\begin{array}{l}\mathrm{CT} \\
\mathrm{TT}\end{array}$ & $\begin{array}{l}2224 \\
1820\end{array}$ & $\begin{array}{l}38.5 \\
68.0\end{array}$ & $\begin{array}{l}1.0 \\
1.0\end{array}$ & $\begin{array}{r}174.2 \\
99.0\end{array}$ \\
\hline $\begin{array}{l}\text { CYP3A4 } \\
{[\mathrm{A}-392 \mathrm{G}]}\end{array}$ & 8 & $\begin{array}{l}\text { AG- } \\
A G\end{array}$ & 1366 & 57.6 & 1.0 & 70.2 \\
\hline $\begin{array}{l}\text { en } \\
\text { CYP3A5 }\end{array}$ & 7 & $\begin{array}{l}A A- \\
A G\end{array}$ & 1167 & 67.0 & 1.0 & 53.3 \\
\hline [A6986G] & 22 & $\begin{array}{l}\mathrm{AA-} \\
\mathrm{GG}\end{array}$ & 2955 & 37.5 & 1.0 & 195.8 \\
\hline
\end{tabular}

Conclusions: Possibly due to the low number of patients a tendency but no significant difference was found between the MDR-1 genotypes. Significant effect on the tacrolimus pharmacokinetics was seen for the CYP3A genotypes.
1 Hesselink DA, et al. Clin Pharmacol Ther 2003; 74: 245-54.

\section{A reanalysis of two studies that reported contrasting results on the association between statin use and risk of fracture in the same data source}

F. de Vries, ${ }^{1,2}$ C. S. de Vries, ${ }^{2}$ C. Cooper, ${ }^{3}$ H. G. M. Leufkens, ${ }^{1}$

\& T.-P. van Staa ${ }^{1,2,3}$

'Utrecht Institute for Pharmaceutical Sciences, Utrecht University,

Utrecht, The Netherlands, 'Postgraduate Medical School,

University of Surrey, Guildford, United Kingdom, ${ }^{3} \mathrm{MRC}$

Epidemiology Resource Centre, University of Southampton,

Southampton General Hospital, Southampton, United Kingdom

Background: In 2000 and 2001, two case-control studies were independently conducted to evaluate the association between use of statins and risk of fracture, and published in the leading clinical journal JAMA. Both studies used the same study data source, the UK General Practice Research Database (GPRD), but found opposite results. The objective of this study was to evaluate the methodological reason for this.

Methods: We repeated both study designs in GPRD: a 'nested' casecontrol dataset, with fracture cases matched to controls nested within a selected cohort, and a 'population-based' case-control dataset, with both cases and controls sampled from the total GPRD population. The cases and controls were matched by gender, age (5-year band) and general practice.

Results: The study included 131,855 fracture cases. In the 'nested' case-control dataset, only $37 \%$ of the cases were matched by year of birth, while this was $99 \%$ in the 'population-based' dataset. The crude OR for hip fracture in statin users differed between the two designs $(0.37$ [95\% CI $0.27-0.52$ ] in the 'nested' and 0.54 [95\% CI $0.39-0.74]$ in the 'population-based' dataset). But this difference reduced when matching by year of birth, rather than by 5 -year band (crude ORs 0.58 [95\% CI 0.43-0.79] and 0.61 [95\% CI 0.44-0.88], respectively). Other factors that introduced differences included the exposure time-window, confounders selection and exclusion of highrisk patients. It was found that the risk of hip fracture was reduced after already one statin prescription (30 days of treatment) and remained stable with increased use.

Conclusion: Choices in study design may lead to contrasting results. A detailed sensitivity analysis may be important in the study of health care database.

\section{Measured mycophenolic acid concentrations after administration of mycophenolate sodium (Myfortic(8) are higher with EMIT compared to HPLC}

B. C. M. de Winter, ${ }^{1}$ R. M. van Hest, ${ }^{1}$ L. B. Hilbrands, ${ }^{2}$

A. G. Vulto, ${ }^{1}$ T. van Gelder ${ }^{1}$ \& R. A. A. Mathot ${ }^{1}$

'Dept of Hospital Pharmacy, Erasmus MC, Rotterdam, and 2Dept of Nephrology, University MC Nijmegen, The Netherlands

Background: Most renal transplantation recipients receive mycophenolic acid (MPA) to prevent rejection. Currently available preparations are mycophenolate mofetil (MMF, Cellcept $\left.{ }^{\circledR}\right)$ and enteric coated mycophenolate sodium (EC-MPS, Myfortic $\left.{ }^{\circledR}\right)$. The concentration of MPA in human plasma can be measured by HPLC or immunoassay (EMIT). MPA concentrations resulting from MMF administration, measured with EMIT are structurally higher compared to HPLC, probably as a result of cross-reactivity of the antibody used in the EMIT assay with the acyl glucuronide metabolite (Ac-MPAG). Due to the formulation of EC-MPS the release of MPA into the small intestine is delayed. This may influence the ratio of MPA to Ac-MPAG during a dosing interval. This study compares EMIT measurements of MPA after EC-MPS administration with HPLC measurements, in order to determine whether a bias also exists for EC-MPS.

Methods: A total of 78 MPA plasma samples from 7 kidney transplant patients, obtained throughout 12-hr dosing intervals of ECMPS were measured by EMIT and a validated HPLC method. Results from the EMIT assay were compared with those of the HPLC method, and bias and precision were calculated. 
Results: The EMIT assay significantly $(\mathrm{p}<0.05)$ overestimated MPA concentrations compared to HPLC (fig. 1): in samples with a concentration below $3.5 \mathrm{mg} / \mathrm{L}(\mathrm{n}=58)$ the mean MPA concentration was $1.36 \mathrm{mg} / \mathrm{L}$ (HPLC) vs. $1.65 \mathrm{mg} / \mathrm{L}$ (EMIT) (mean overestimation:

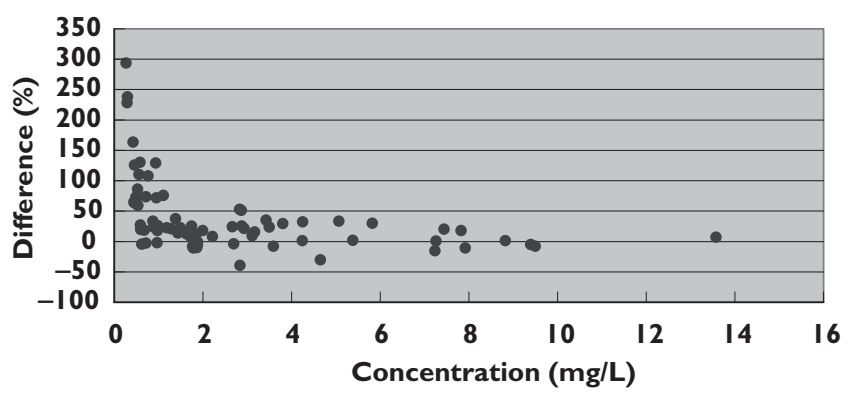

Figure 1

Difference of MPA concentration measured by EMIT in comparison with HPLC
44.5\%; 95\% CI: 27.6-61.5\%; precision: $77.9 \%$; 95\% CI: $45.5-100.3 \%)$. For all other samples $(n=20)$ with concentrations above $3.5 \mathrm{mg} / \mathrm{L}$ (mean: $6.25 \mathrm{vs} .6 .75 \mathrm{mg} / \mathrm{L}$ ) average overestimation was $13.0 \%$ (95\% CI: 7.1-18.9\%; precision: $25.5 \%$; $95 \%$ CI: $21.0-29.4 \%)$

Discussion: In parallel with the overestimation of MPA concentration by EMIT after MMF administration $(0.29 \mathrm{mg} / \mathrm{L}$ in the concentration range $0-2 \mathrm{mg} / \mathrm{L}^{1}$ ), the bias with EC-MPS is probably caused by cross-reactivity of the antibody used in the EMIT assay with AcMPAG. Consequently, EMIT results may not solely represent MPA concentration. Since Ac-MPAG has immunosuppressive activity, EMIT results may reflect the overall immunosuppressive activity of EC-MPS more complete than HPLC.

Conclusion: MPA concentrations resulting from EC-MPS administration are overestimated by the EMIT assay in comparison with HPLC. Whether this bias hinders therapeutic drug monitoring needs to be investigated, but seems unlikely.

\section{Dade Behring; Emit®2000 Mycophenolic Acid Assay.}

\section{Effects of utilization of computer based teaching (CBT) programs on test performance: the TRC experience}

K. L. Franson', E. A. Dubois ${ }^{2}$ \& A. F. Cohen'

'Centre for Human Drug Research, Leiden; 'Leiden University Medical Center

Background: The Teaching Resource Centre (TRC) began to provide pharmacology education to medical students at a time when there was no formalized pharmacology education in the curriculum. The TRC created CBT that linked pharmacology to (patho)-physiology, and provided students with formative feedback. Since using TRC CBT is not compulsory, a back-end was built into the CBT programs to assess student use.

Aim: To demonstrate an increase in student's test grades as a measure that students derive benefit from using TRC CBT.

Methods: CBT utilization was assessed using log-in data from programs such Blackboard ${ }^{\circledR}$, 'Lesson Registration System', and the backend of the TRC CBT. CBT utilization parameters included the number of CBT's used by a student in a course and time spent using the CBT program. Student performance was assessed by the final grade in a course ( 1 to 10 system and $>5$ is needed to pass), and the score on the pharmacology section of the final ( 5 or 7 points, approximately $10 \%$ of final).

Data analysis: The number of CBT's used was determined by recording only the action of logging in. Student ID's that were used for logging in but did not appear on the exam were deleted. Time spent using CBT's was determined from more than 80,000 hits to TRC CBT's by more than 900 students. Time spent on one topic was cut-off when it was $<5$ or $>$ the 95 percentile. Student ID's that did not appear on the exam list were deleted. Students not showing up to the test were given 0 .

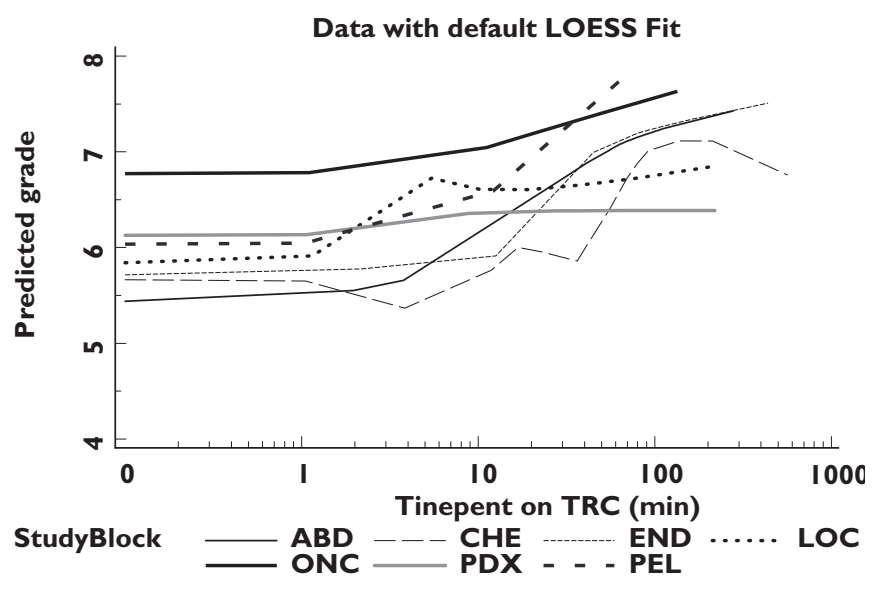

Results: Grade regression of time spent using TRC CBT's

The confounding variable of inter-student performance variability cannot be separated from the above graph. Yet, a repeated measures analysis to calculate the grade regression on time spent for each student demonstrated an intercept of 6.6 and a slope of 0.0031 for all courses. The plausibility of reward bias was eliminated by evaluating the correlation factor between the grade regression for the final grades versus the pharmacology section grades $(\mathrm{P}=0.8839)$.

Conclusions: Determining the effectiveness of a CBT intervention is difficult. However, increased TRC CBT use by individual students leads to a (small) increase in their grades. Analyzing CBT utilization and grade data can foster improvements in CBT programs. 


\section{Changes in medication in relation to epilepsy-related hospitalization: a case cross-over study}

K. B. Handoko, ${ }^{1,2}$ W. A. J. J. Hermens, ${ }^{2}$ J. E. F. Zwart-van Rijkom, ${ }^{2}$

P. C. Souverein' \& A. C. G. Egberts'

'Department of Pharmacoepidemiology and Pharmacotherapy,

Utrecht Institute of Pharmaceutical Sciences, Utrecht,

Netherlands and 'Hospital Pharmacy, 'Ziekenhuisapotheek

Noord-oost Brabant', 's-Hertogenbosch, Netherlands

Background: In patients treated for epilepsy a delicate balance in therapy exists (Sander, 2004; Brodie \& French, 2000). Altering medication, both non-antiepileptic as well as antiepileptic therapy, may lead to a decreased effectiveness and possibly an increased seizure risk.

Aims: To determine whether changes in medication are associated with epilepsy-related hospital admissions.

Methods: Data for this study were obtained from the PHARMO Record Linkage System and covered the period of January 1998 to December 2002. We conducted a case cross-over study among patients with a first epilepsy related hospital admission who had used at least one antiepileptic drug (AED) continuously in a 200-day time window before the admission date. Within these patients, changes in a 28-day window before hospitalisation were compared with changes in the 28-day windows 6,12,18 and 24 weeks before hospitalisation. Two types of changes in medication were defined. Firstly, we assessed changes in non-antiepileptic therapy, i.e. introduction of new drugs. Secondly, we evaluated changes in AED therapy, subdivided into intentional changes (add-on,discontinuation, switch of AEDs and changes in AED dose) and non-intentional changes (introduction or discontinuation of drugs that could effect the plasma concentration of AEDs). Conditional logistic regression analysis was used to calculate the Odds Ratios (OR) and 95\% Confidence Intervals (CI).

Results: Of 1,185 patients with 1,628 epilepsy-related hospitalisations, 217 patients met the inclusion criteria for our study. Introduction of three or more non-antiepileptic drugs was significantly associated with epilepsy-related hospitalisations (OR: 4.80; 95\% CI: 2.12-10.87). No significant association was found between changes in AED therapy and epilepsy-related hospitalisation. Add-on of AEDs tended to be associated with a reduced risk (OR: 0.46, 95\% CI: 0.14-1.54), while discontinuation of AEDs showed a trend towards an increased risk (OR: 2.57, 95\% CI: 0.81-8.17). Introduction or discontinuation of drugs that could effect the AED plasma concentration rarely occurred and no ORs could be calculated.

Conclusions: In our study we showed that starting three or more new drugs leads to a nearly five times higher risk for epilepsy-related hospital admissions. Changes in AED therapy were not significantly associated with epilepsy-related hospitalisations.

Sander JW. The use of antiepileptic drugs-principles and practice. Epilepsia 2004; 45 Suppl 6: 28-34.

Brodie MJ, French JA. Management of epilepsy in adolescents and adults. Lancet 2000; 356: 323-9.

\section{Patient counselling by community pharmacists at discharge from hospital}

J. G. Hugtenburg, ' J. J. Beckeringh ${ }^{2} \&$ P. E. De Jong ${ }^{3}$

'VU University medical center, Department of Medical

Pharmacology, Amsterdam, ${ }^{2}$ Dutch College of Insurance

Companies, Diemen ${ }^{3}$ Association of Amsterdam Community

Pharmacists, Amsterdam

In addition to drugs prescribed by specialists, most patients with chronic diseases also use drugs prescribed by general practitioners. A stay in a hospital often leads to changes in the drug regimens of these generally elderly patients. At discharge for many patients it is unclear which drugs have to be used. Since in the Netherlands drugs at discharge are usually supplied by community pharmacists, it is also their responsibility that all changes are properly implemented and that patients are adequately informed about the drugs they should use after discharge. In order to optimise existing services, the Association of Amsterdam Community Pharmacists recently developed a comprehensive pharmaceutical care protocol for patient counselling at discharge from hospital.

Aims: To evaluate the protocol and to study its effects on pharmacotherapy.

Methods: A controlled study involving 37 Amsterdam community pharmacies (45\% of total; 19 intervention and 18 control). All patients taking five or more prescribed medications at discharge were eligible. Each pharmacy contributed maximally 20 patients in subsequent order. Upon or shortly after delivery of their discharge medication patients of intervention pharmacies were visited by their pharmacist athome (aim) or received education in the pharmacy. All patients received a written outline of their rationalised drug regimen. If possible home supplies of drugs were checked and rationalised. Patients attending control pharmacies received normal services.

A questionnaire study after patients' satisfaction with drug counselling was also performed.

Results: Patient counselling was received by 336 patients by 19 pharmacists (intervention group) at home (65\%), in the pharmacy (20\%) or by telephone $(15 \%) .40 \%$ of the patients asked questions about their drugs or raised drug problems. In the control group, 379 patients, questions raised by patients resulted in counselling with 38 patients $(10 \%)$. In the intervention and control group $69.6 \%$ and $39.4 \%$ of the patients received a smaller number of drugs than prescribed by the hospital and $11.1 \%$ and $1.1 \%$ received additional drugs. In the intervention and control group $28.9 \%$ and $5.8 \%$ of the patients received a smaller amount of a drug dispensed and in $13.4 \%$ and $8.4 \%$ of cases the dose was adapted. The expenses saved by the pharmacy amounted to 17 and 0 euro per patient of the intervention and the control group. After 15 months $15.5 \%$ and $19 \%$ of patients of the intervention and control group have died. 87\% (intervention) and $50 \%$ (control) of patients were satisfied with counselling by their pharmacists.

Conclusion: Patient counselling at discharge from hospital is a meaningful pharmaceutical care activity of pharmacists. Structured patient counselling resulted in more attention of the pharmacists for drug use of these chronically ill patients. Patient counselling at discharge from hospital increased patients' satisfaction. 


\section{Lack of analgesic effect form topical morphine in painful arterial leg ulcers}

M. M. P. M. Jansen, ${ }^{1,2}$ J. C. van der Horst, ${ }^{3}$ P. G. M. van der Valk, ${ }^{4}$ P. F. M. Kuks, Z. Zylicz, ${ }_{1}^{5}$ \& A. A. van Sorge ${ }^{2}$

'Midden-Brabant Hospital Pharmacy, Tilburg, Netherlands;

2Hospital Pharmacy, Rijnstate Hospital, Arnhem, Netherlands;

${ }^{3}$ Dept. of Dermatology, Rijnstate Hospital, Arnhem, Netherlands;

${ }^{4}$ Dept. of Dermatology UMC St. Radboud, Nijmegen,

Netherlands; ${ }^{5}$ Chair and Dept. of Palliative Care, Mikolay

Kopernik University, Collegium Medicum, Bydgoszcz, Poland

Aims: Arterial ulcers are known to be very painful. Even with current knowledge appropriate analgesia is often problematic. ${ }^{1}$ We examined whether analgesia in arterial ulcers can be obtained by topical application of 1 gram of a temperature-sensitive hydrogel containing $0.5 \% \mathrm{~m} / \mathrm{m}$ of morphine. $\mathrm{HCl}$.

Methods: 9 patients with a painful arterial ulcer were included in the study. The pain intensity at inclusion was at least 5 on a 10 point Numeric Rating Scale. Patients were treated in a double-blind, placebo controlled, 3 -way crossover design. The 3 treatments consisted of morphine hydrogel in parallel with placebo subcutaneous infusion (A), placebo gel in parallel with subcutaneous infusion of $5 \mathrm{mg}$ morphine over 6 hours (B) and placebo gel in parallel with placebo subcutaneous infusion (C). Treatment was started between 8 and 9 am. Pain was assessed during the first 12 hours after application of the hydrogel and start of the subcutaneous infusion. Results were analysed with a mixed linear model for repeated measurements with one fixed factor and one random intercept.

Results: The mean pain score (SE) at inclusion was 6.3(0.6). During treatment the mean pain scores were 5.2(0.6), 4.8(0.8) and 5.3(0.7) for treatment A, B and C, respectively. For each treatment the pain

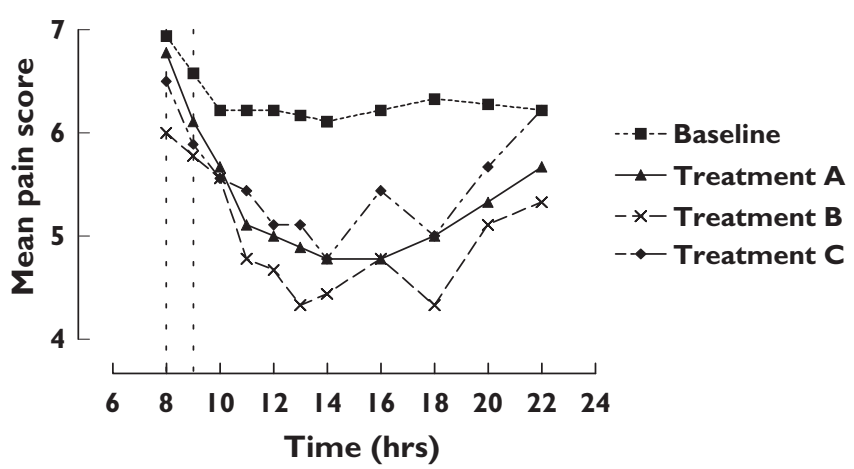

Figure 1

scores differed significantly from baseline (estimated difference (SE): $1.1(0.3), 1.4(0.3)$ and $0.9(0.3)$ with $\mathrm{p}<0.012, \mathrm{p}<0.002$ and $\mathrm{p}<0.024$ respectively). Figure 1 shows the mean pain score of all patients during baseline and treatments. However, these results were not clinically relevant. There was no difference between the 3 treatments.

Conclusion: This study showed no clinically relevant analgesic effect of topical application of morphine in patients with painful arterial leg ulcers. The absence of this effect might be due to the neuropathic and/or ischemic component in this type of pain and/or lack of inflammation and/or chronic nature of the ulcer and therefore absence of available and functional opioid receptors at the site of the ulcer. ${ }^{2}$ Further investigation is highly recommended.

1 Cooper et al. International Journal of Lower Extremity

Wounds. 2003; 2: 189-97.

2 Bigliardi et al. J Invest Dermatology 2003; 120: 145-52.

\section{Classification and occurrence of clinically significant drug-interactions with 5-fluorouracil/leucovorin in patients with colorectal cancer}

\author{
F. G. A. Jansman, 1,2 J. A. Jansen, ${ }^{2}$ J. L. L. M. Coenen, ${ }^{3}$ \\ J. C. De Graaf, ${ }^{3}$ W. M. Smit, ${ }^{4}$ D. Th. Sleijfer ${ }^{5}$ \& \\ J. R. B. J. Brouwers ${ }^{2}$ \\ 'Department of Clinical Pharmacy, Isala klinieken, Zwolle, \\ ${ }^{2}$ Groningen University Institute for Drug Exploration, Groningen, \\ ${ }^{3}$ Department of Medical Oncology, Isala klinieken, Zwolle, \\ ${ }^{4}$ Department of Medical Oncology, Medisch Spectrum Twente, \\ Enschede, ${ }^{5}$ Department of Medical Oncology, UMC Groningen, \\ the Netherlands
}

Aims: Interactions between anticancer drugs and other drugs may influence efficacy and toxicity of the anticancer treatment. The aims of the study were to assess the clinical significance of drug-interactions with 5-fluorouracil (5-FU), and to examine the occurrence of these interactions in 122 colorectal cancer patients who were treated with 5-FU/leuovorin.

Methods: Literature was searched for drug-interactions with 5-FU. These interactions were classified for documentation evidence and severity level as proposed by the standard reference Drug Interaction Facts, assigning 5 levels of clinical significance [1]. Classification was performed by a panel of medical oncologists and clinical pharmacologists. Subsequently, by comparison of all co-medication that was used by the patients, the occurrence of clinical significant interactions with 5-FU was assessed.

In addition, toxicity of 5-FU/leucovorin in these patients was determined according to the NCI-CTCriteria v2.0.

Results: In literature, 17 interactions with 5-FU were found, of which 11 were classified as clinically significant by the panel members. Agreement of classification results between panel members was substantial (mean $\kappa=0.70$ ). Patients in this study used 4,7 different drugs, apart from 5-FU/leucovorin. Analysis of the co-medication of all patients revealed possible clinically significant interactions in 4 patients, who used hydrochlorothiazide ( 2 patients) or folic acid (2 patients). Toxicity percentages of 5-FU/leucovorin in 122 colorectal cancer patients corresponded with percentages from literature.

Conclusion: From literature, 11 clinically significant interactions with 5-FU could be identified, according to the classification method from Drug Interaction Facts [1]. The occurrence of these interactions in the prospectively studied colorectal cancer patients, was low. However, medical oncologists and clinical pharmacists should have regard for these interactions in daily practice. When applicable, the clinical significant interactions can also be recommended to be added to interactions-databases that are used for medication-surveillance.

1 Tatro DS. Drug interaction facts. St Louis: Facts and Comparisons, 2004. 


\section{Determinants of the elimination of methotrexate and 7-hydroxy-methotrexate following high-dose infusional methotrexate}

M. Joerger ${ }^{1,2}$, A. D. R. Huitema', H. J. G. D. van den Bongard ${ }^{3}$,


'Dep of Pharmacy \& Pharmacology, Slotervaart Hospital, Amsterdam, NL ${ }^{2}$ Dep of Medical Oncology, The Netherlands Cancer Institute, Amsterdam NL ${ }^{3}$ Dep of Radiotherapy, The Netherlands Cancer Institute, Amsterdam, NL ${ }^{4}$ Dep of Biomed Analysis, Faculty of Pharmaceut Sciences, Utrecht University, NL

Introduction: The identification of methotrexate (MTX) and 7hydroxy-methotrexate (7-OH-MTX) pharmacokinetics and its determinants is very useful in providing guidelines to prevent potentially fatal nephrotoxicity from high-dose MTX (HDMTX) schedules. MTX is prone to drug interactions, particularly synergistic nephrotoxicity with non-steroidal anti-inflammatory drugs (NSAIDs), benzimidazoles, penicillin and sulphonamides among others.

Methods: Routine 24- and 48-hr blood samples were collected in 76 patients after having received a total of 309 courses of HDMTX (dose range $300 \mathrm{mg} / \mathrm{m}^{2}$ to $12 \mathrm{~g} / \mathrm{m}^{2}$ ). Intensive sampling was available from 21 patients. Leucovorin rescue was started $24 \mathrm{hrs}$ after the start of MTX and continued dependent on MTX plasma levels. MTX and 7-OH-MTX concentration-time data were subjected to a population pharmacokinetic and covariate analysis using nonlinear mixed-effect modeling (NONMEM).

Results: Treatment-related mortality was $1.3 \%$ (1 patient with fatal renal failure). 2 Patients had markedly increased exposure to MTX (figure 1). A three-compartment model best described the concentration-time data of MTX, while a two-compartment model best described the concentration-time data of 7-OH-MTX. Clearance (CL) was $8.85 \mathrm{~L} / \mathrm{hr}$ for MTX and $2 \mathrm{~L} / \mathrm{hr}$ for $7-\mathrm{OH}-\mathrm{MTX}$. Baseline creatinine clearance correlated with $\mathrm{CL}_{\mathrm{MTX}}$ and $\mathrm{CL}_{7 \text {-OH-MTX }}$. Concurrent administration of benzimidazoles led to a $27 \%$ reduction of $\mathrm{CL}_{\text {MTX }}$ and a $39 \%$ reduction of $\mathrm{CL}_{7 \text {-он-мтх }}$. Prior administration of NSAIDs led to a $16 \%$ reduction of $\mathrm{CL}_{\mathrm{MTX}}$ and a $38 \%$ reduction

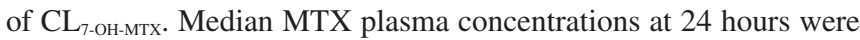
higher in patients with concurrent benzimidazoles compared to patients without benzimidazoles, as were median MTX plasma concentrations at 48 hours in patients with concurrent benzimidazoles compared to patients without benzimidazoles. Median MTX plasma

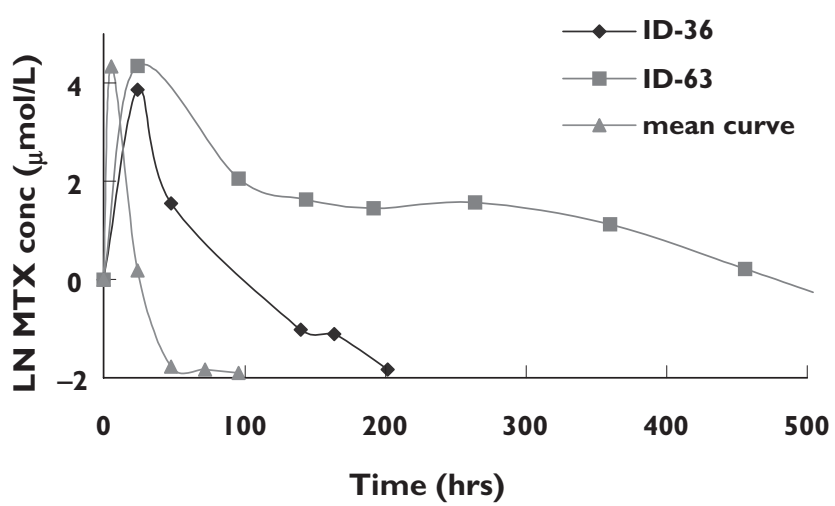

Figure 1

Table 1

\begin{tabular}{|c|c|c|c|c|}
\hline \multirow[b]{2}{*}{$\begin{array}{l}\text { Patient } \\
\text { subgroup }\end{array}$} & \multicolumn{2}{|c|}{$\begin{array}{l}\text { Median } 24 \mathrm{hr} \\
\text { conc }(\mu \mathrm{mol} / \mathrm{L})\end{array}$} & \multicolumn{2}{|c|}{$\begin{array}{l}\text { Median } 48 \mathrm{hr} \\
\text { conc }(\mu \mathrm{mol} / \mathrm{L})\end{array}$} \\
\hline & MTX & $\begin{array}{l}\text { 7-OH- } \\
\text { MTX }\end{array}$ & MTX & $\begin{array}{l}\text { 7-OH- } \\
\text { MTX }\end{array}$ \\
\hline $\begin{array}{l}\text { + benzimidazoles } \\
\text { no benzimidazoles } \\
\text { p-value }\end{array}$ & $\begin{array}{l}2.79 \\
0.63 \\
<10^{-3}\end{array}$ & $\begin{array}{l}5.22 \\
3.89 \\
<10^{-3}\end{array}$ & $\begin{array}{l}0.21 \\
0.08 \\
<10^{-3}\end{array}$ & $\begin{array}{l}1.46 \\
0.89 \\
<10^{-3}\end{array}$ \\
\hline $\begin{array}{l}\text { prior NSAIDs } \\
\text { no NSAIDs } \\
\text { p-value }\end{array}$ & $\begin{array}{l}1.68 \\
0.67 \\
<10^{-3}\end{array}$ & $\begin{array}{l}4.25 \\
3.98 \\
0.91\end{array}$ & $\begin{array}{l}0.12 \\
0.08 \\
<10^{-2}\end{array}$ & $\begin{array}{l}1.32 \\
0.895 \\
<10^{-3}\end{array}$ \\
\hline
\end{tabular}

concentrations were also higher in patients with prior NSAIDs compared to patients without prior NSAIDs (table 1).

Conclusion: In patients receiving HDMTX, concurrent administration of benzimidazoles led to a significant decrease of $\mathrm{CL}_{\mathrm{MTX}}$ and $\mathrm{CL}_{\text {7-Он-мтх }}$, comparable to what has been found in patients with prior administration of NSAIDs. The data suggest that benzimidazoles should be seen as a relative contraindication for HDMTX.

\section{The beta-blocker nebivolol and exercise-capacity in patients with mild hypertension}

\section{W. Mäkel, ${ }^{1}$ R. Agrawal, ${ }^{2}$ A. Lichtenthal ${ }^{2}$ \& T. J. Cleophas ${ }^{3}$ \\ 'Clinical Research Facilities, Schaijk, Netherlands, ${ }^{2}$ Berlin- \\ Chemie, Berlin, Germany, ${ }^{3}$ European Interuniversity College \\ Pharmaceutical Medicine, Lyon, France}

Background: The effect of beta-blockers on exercise-capacity in patients with hypertension has not been systematically studied, and is relevant because alternative treatments are available

Objective: To investigate the effect of the third generation betablocker nebivolol on exercise-capacity in a large and representative number of patients with mild hypertension. The Nationwide Efficacy-safety Nebivolol Surveillance Study ${ }^{1}$ enabled us to do so.
Methods: 6357 mildly hypertensive non-claudicants were assessed for exercise-capacity indicated by the distance a man could walk on the flat at normal pace. ${ }^{2}$ Three categories were defined: (1) <100 m, (2) 100-200 m, (3) >300 m. Patients were evaluated prior to, after 3 and after 6 weeks of nebivolol treatment $5-10 \mathrm{mg}$ daily.

Results: At baseline exercise-capacity was limited in $18.3 \%$ of the patients without and in $32.5-53.1 \%$ of the patients with cardiopulmonary comorbidities. After nebivolol treatment exercise-capacity increased by at least one category in $14.4 \%$ of the patients without and in $25.1-36.5 \%$ of the patients with cardiopulmonary comorbidities ( $p<0.01$ to $\mathrm{p}<0.001$ versus baseline). After adjustment for baseline the improvements between patients with and without comorbidities were not significantly different from one another. 
Conclusions: Not only hypertensive patients with cardiopulmonary comorbidities, but also those without have a reduced exercise capacity. In the latter group this is possibly due to peripheral vasoconstriction. The beta-blocker nebivolol improved this reduced exercise-capacity. The improvement was largely independent of car- diopulmonary comorbidities, and is possibly due to nebivolol's vasodilatory effects.

1 Cleophas TJ, et al. Am J Ther 2005; 10: 205.



\section{Valproic acid-induced metabolic encephalopathy, a life threatening complication a case report and pathofysiological mechanisms}

M. C. Meinardi, G. B. van den Berg, A. Groenhuijzen, H. Maas,

A. C. G. Egberts \& F. H. J. Wolfhagen

Twee Steden hospital, Tilburg

A 62-year-old woman presented with fever and drowsiness. She had a history of atrial fibrillation complicated by a stroke. Her medication comprised sotalol, paroxetine, duphhalac, prednison, ASA, omeprazol, actonel, baclofen and valproic acid. Physical examination revealed a somnolent woman, with no other abnormalities. A chest X-ray showed a rightsided infiltrate. She was admitted and treated with cefuroxim. Initially elevated infection parameters and liver enzymes normalised. However patients consciousness decreased progressively and food intake was low. On day 7 feeding by nasogastric tube was started. At the 10th day of admittance, she became comatose (EMV-score 3) and needed artificial ventilation. Her ammonia level appeared to be $286 \mu \mathrm{mol} / \mathrm{l}$ (normal range: 10 $45 \mu \mathrm{mol} / \mathrm{l})$ with normal liver enzyme tests. As the hyperammonaemia was thought to be caused by valproic acid, this drug was discontinued. After 3 days, ammonia levels fell, patient regained consciousness and detubation was possible. Screening for ornithine transcarbamoylase gene mutations, deletions and polymorfisms was negative.

Discussion: Isolated hyperammonaemia without liver insufficiency is reported as a side effect of valproic acid use. Valproic acid can inhibit conversion of ammonia to urea in the liver and elevate renal ammonia production.
Primary hyperammonaemia can be caused by an inborn error of metabolism with diminished ornithine transcarbamoylase activity. Individuals with this enzyme deficiency are especially susceptible to this complication.

Secondary hyperammonaemia is due to a deficit of essential substrates or co-factors needed for the urea cycle such as carnitine. Valproic acid binds carnitine, upon which it is renally secreted. Also in the kidney, glutaminase activity is increased by valproic acid and glutamine is thus converted in glutamate and ammonia.

Intracerebrally, astrocyte glutamine production is stimulated due to elevated ammonia, eventually resulting in swelling of the astrocyte, which gives a loss of functionality.

Furthermore, neurosteroids and peripheral benzodiazepine receptors are increased. These effects cause $\mathrm{GABA}_{\mathrm{A}}$ system overactivation, leading to neuronal dysfunction and neuroinhibition.

We postulate that the somnolence at the time of admittance was caused by an elevated ammonia level due to the use of valproic acid. The use of baclofen might have played a role because of the additional stimulating effect on the GABA system. Furthermore nasogastric tube feeding might have led to a higher protein load with an additional elevation of ammonia and worsening the metabolic encephalopathy.

Conclusion: In patients using valproic acid an impaired level of consciousness should raise the suspicion of valproic acid-induced metabolic encephalopathy, even in the absence of elevated liver enzymes. Replacement of valproic acid is the treatment of choice and was in our case life-saving.

\section{Potassium channel blockade restores the attenuated noradrenaline sensitivity in human endotoxemia}

P. Pickkers, ${ }^{1,2}$ M. J. Dorresteijn, ${ }^{2}$ P. Smits, ${ }^{1}$ H. van der Hoeven ${ }^{2}$

Departments of 'Pharmacology-Toxicology and 'Intensive Care

Medicine, Radboud University Nijmegen Medical Centre, PObox

9101, 6500HB Nijmegen, The Netherlands

Introduction: Vasodilatory shock is a major problem in intensive care medicine. It is recently recognized that activation of vascular potassium $(\mathrm{K})$ channels may play an important role in sepsis-induced vasodilation and attenuated sensitivity to noradrenaline. We examined whether endotoxin administration to healthy volunteers resulted in an attenuated noradrenaline sensitivity and whether this effect could be restored by K-channel blocker tetra-ethyl ammonium (TEA).

Methods: Human volunteers received 2 ng/kg E. Coli endotoxin. The brachial artery was cannulated for infusion of drugs. Forearm blood flow (FBF) was measured using venous occlusion plethysmography. Noradrenaline was administrated intra-arterially at 1,3,10,30 ng/ $\mathrm{min} / \mathrm{dl}$ and the vasoconstrictive response to noradrenaline was deter- mined before and $4 \mathrm{hrs}$ after endotoxin was administrated in the presence or absence of TEA $(1 \mathrm{mg} / \mathrm{min} / \mathrm{dl})$. Data is expressed as mean \pm SEM. Differences were tested by ANOVA repeated measures or Student-t, as appropriate. A p-value $<0.05$ was considered to indicate significance.

Results: Endotoxin administration induced the expected flu like symptoms and fever (max. temp. $38.3 \pm 0.1^{\circ} \mathrm{C}, \mathrm{p}<0.001$ ). Mean arterial pressure decreased from $93 \pm 2$ to $79 \pm 2 \mathrm{mmHg}(\mathrm{p}<0.001)$ and heart rate increased from $60 \pm 2$ to $95 \pm 2 \mathrm{bpm}(\mathrm{p}<0.001)$.

Intra-arterial noradrenaline infusion decreased FBF: Percentage of baseline ratio (infused/noninfused arm) $100 \pm 0,84 \pm 4,70 \pm 4,55$ $\pm 4,38 \pm 4 \%$.

Following endotoxin administration, the noradrenaline-induced vasoconstriction was attenuated: $100 \pm 0,101 \pm 4,92 \pm 4,83 \pm 6$, $56 \pm 7 \%(\mathrm{p}<0.001$, pooled data, $\mathrm{n}=25)$. Time control experiments $(n=6)$ demonstrated excellent repeatability of the attenuated noradrenaline response after the administration of endotoxin (see figure, left). Intra-arterial infusion of $\mathrm{K}$ channel blocker TEA almost completely restored the vasoconstrictive effect of noradrenaline (see figure, right, $\mathrm{n}=6, \mathrm{p}=0.045$ ). 
\%FBF

(ratio infused/non-infused arm)

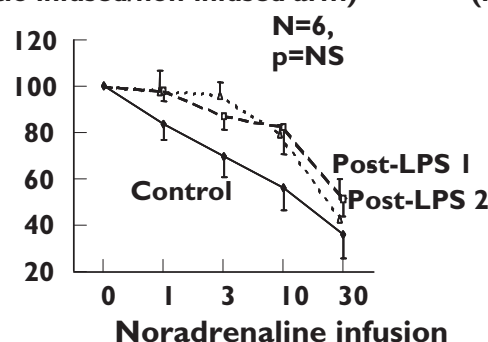

\%FBF

(ratio infused/non-infused arm)



Conclusions: In experimental human endotoxemia, noradrenaline sensitivity is decreased. $\mathrm{K}$ channel blocker TEA almost completely restores the vasoconstrictive effects of noradrenaline. Our study demonstrates that endotoxin-induced vascular $\mathrm{K}$ channel activation plays a major role in the observed attenuated sensitivity to noradrenaline during human endotoxemia.

\section{Effects of a contraceptive vaginal ring delivering nestorone ${ }^{\circledR}$ and ethinyl estradiol, and a combined oral contraceptive containing levonorgestrel, on markers of arterial disease}

M. Rad, ${ }^{1}$ R. Sitruk-Ware, ${ }^{2}$ C. Kluft, ${ }^{3}$ M. L. de Kam, ${ }^{1}$ I. Sivin, ${ }^{2}$

J. Menard, ${ }^{4}$ \& J. Burggraaf'

'Centre for Human Drug Research, Leiden, the Netherlands

${ }^{2}$ Center for Biomedical Research, Population Council, New York, USA ${ }^{3}$ TNO Quality of Life, Gaubius Laboratory, Leiden, the Netherlands ${ }^{4}$ INSERM Unit 367, Paris, France

Introduction: Combined oral contraceptives (COCs) alter biomarkers associated with risk of arterial disease in healthy individuals. To evaluate effects of non-oral delivery, we compared a contraceptive vaginal ring (CVR) daily delivering $150 \mu \mathrm{g}$ Nestorone $^{\circledR}$ (NES), a non-androgenic progestin, and $15 \mu \mathrm{g}$ ethinyl estradiol (EE), with a second-generation COC containing $150 \mu \mathrm{g}$ levonorgestrel (LNG), an androgenic progestin, and $30 \mu \mathrm{g}$ EE per daily tablet.

Methods: In a parallel, open-label study, 45 healthy women (18-34 years) were randomized to use the CVR $(n=23)$ or COC $(n=22)$. Fasting blood was collected before and after 3 cycles of treatment.
Assays included angiotensinogen (AI), CRP, fibrinogen, D-dimer, total cholesterol (TC), HDL, LDL and triglycerides (TG).

Analysis: CVR effects were compared to those of COC using ANCOVA with baseline values as co-variates, yielding a $95 \% \mathrm{CI}$ of $\%$ treatment difference, reported below for each variable.

Results: The CVR elevated CRP (16-275\%) more than the COC, while the AI, fibrinogen and D-dimer increased equally with both treatments. HDL was increased in CVR and decreased in COC users, while LDL decreased in CVR and increased in COC users, resulting in treatment differences $19-47 \%$ and $-33--12 \%$, respectively. TC was unchanged, while the COC elevated the TC/HDL ratio $(-28-$ $-14 \%$ ). Although both treatments elevated TG, it was affected more markedly by the CVR (14-69\%).

Conclusion: The observed differences between the NES/EE CVR and the LNG/EE COC effects are most likely due to the difference in androgenicity of the progestins. In contrast to the CVR, the COC has an untoward effect on lipid metabolism. As the elevation in CRP may be related to either inflammatory processes or to a direct stimulatory effect from EE on the liver no definite conclusion can be drawn on the basis of surrogate markers. The net effect of CVR use on longterm arterial risk can be determined only by prospective studies.

\section{Multiple-dose tolerability, safety, pharmacokinetics, and pharmacodynamics of the urotensin-II receptor antagonist palosuran in healthy human subjects}

P. N. Sidharta, ${ }^{1}$ P. L. M. van Giersbergen, ${ }^{1}$ D. Schaarschmidt, ${ }^{2}$ \& J. Dingemanse ${ }^{1}$

${ }^{\prime}$ Actelion Pharmaceuticals Ltd, Allschwil, Switzerland; 2 PHAROS

$\mathrm{GmbH}$, Ulm, Germany

Urotensin-II, a cyclic peptide, and its receptor (UT receptor) have been identified as a novel neurohormonal system, which may be involved in diseases such as renal failure, heart failure, and hypertension. Therefore, UT receptor antagonists may have therapeutic applications in these diseases.

This report describes the multiple-dose tolerability, safety, pharmacokinetics, and pharmacodynamics of palosuran, a selective, nonpeptidic, orally active, potent, and competitive antagonist of the human UT receptor. The chosen design was a single centre, double blind, randomized, placebo-controlled, ascending multiple-dose study. Oral doses of $25 \mathrm{mg}, 125 \mathrm{mg}$, and $500 \mathrm{mg}$ were given b.i.d for 6.5 days to sequential groups of 8 subjects ( 6 on active drug, 2 on placebo). Tolerability and safety were assessed by evaluation of adverse events (AEs), physical examination, vital signs, ECG, and clinical laboratory parameters. For pharmacokinetic and pharmacodynamic evaluation of palosuran, venous blood samples were collected during 12 hours on Day 1, before morning study drug intake from Day 2 to Day 6, and during 36 hours on Day 7. Plasma concentrations of palosuran and U-II were determined. In addition, on Day 1 and 7, urine samples were collected over regular intervals for determination of the excretion of sodium, potassium, creatinine, and unchanged palosuran.

No serious AEs or AEs that led to premature discontinuation were reported. AEs were of a mild to moderate nature and included abdominal distension, diarrhoea, fatigue, loose stools, and catheter 
site reaction. No dose relationship could bediscerned for any AE. No clinically relevant changes in vital signs, ECG, and clinical laboratory test parameters were observed. Palosuran was rapidly absorbed after dosing. Its plasma concentration-time profiles were characterized by two peaks at approximately 1 and 4 hours after drug administration (Figure 1).

The apparent terminal half-life was approximately 25 hours. Steady-state concentrations were reached after 4 to 5 days of dosing. Exposure to palosuran after 6.5 days of treatment was consistently greater than on the first day of treatment (the accumulation factor was approximately 2.5 ). With increasing doses, a more than dose proportional increase in $\mathrm{AUC}_{\tau}$ and $\mathrm{C}_{\max }$ was observed. Urinary excretion of unchanged palosuran was less than $3 \%$ of the administered dose. No consistent effect was found on any of the pharmacodynamic variables (U-II plasma levels, urinary excretion of sodium, potassium, and creatinine). In conclusion, palosuran after multiple-dosing is a well tolerated drug in healthy subjects which warrants further investigation in patients.



Time after treatment start (h)

\section{Figure 1}

Plasma concentration-time profile of $125 \mathrm{mg}$ palosuran b.i.d. during 6.5 days (mean \pm SEM, $\mathrm{n}=6$ )

\section{Prescribing for heart failure in Europe: does the country make the difference?}

\section{H. B. Sturm, W. H. van Gilst \& F. M. Haaijer-Ruskamp Clinical Pharmacology, UMCG, Groningen. The Netherlands}

Differences in prescribing patterns for chronic heart failure (CHF) have been demonstrated repeatedly between countries. It is not clear, whether this phenomenon is caused by different patient characteristics or factors related to the country itself, such as health care systems or culture. Objective: To assess the overall impact of countries on prescribing for $\mathrm{CHF}$ while taking patient characteristics into account.

Method: Data of 11062 patients from primary care physicians in 15 European countries have been analyzed. Differences in prescribing were analyzed taking multidrug regimes into account. Regimes consisting of the same number of drugs were compared to each other. The countrys' influence on prescribing was assessed using multinomial logistical models, which correct for patient characteristics (comorbidities, gender, and age).

Results: Prescribing of drug regimes as recommended in guidelines (crude adherence, Figure 1) ranged from $28.1 \%$ in Turkey to $61.8 \%$ in Hungary. When including additional regimes, justified by patients' comorbidities, overall "rational" prescribing (adjusted adherence) increased by $10 \%$, but differences between countries remained similar.

Country is a significant determinant for the number of drugs used (intensity of treatment) and for individual drug regimes. For each drug regime an odds ratio is calculated, indicating its likelihood to be given in each country compared to the reference country (the country with the highest recommended prescribing).

For example in Czech Republic digoxin as single drug therapy was more likely than an ACE-inhibitor (ACE) (OR: 3.45; 95\%CI: 2.56-4.64), while the combination of ACE with digoxin was not significantly more likely than in the reference country Hungary (OR: 1.17; 95\%CI: $0.88-1.55$ ). The multivariate statistical models explained between 35 and $42 \%$ of the total variance (R-square: $0.35-0.42)$, country contributed 7-8\% ( $\mathrm{p}<0.005)$.

Conclusion: Country as such has a clear influence on the number of drugs used and the prescribing of individual drug regimes also when correcting for patient characteristics. The prove of a distinct role of the country underlines the need for a better understanding of how health-care systems and culture affect management of heart failure.

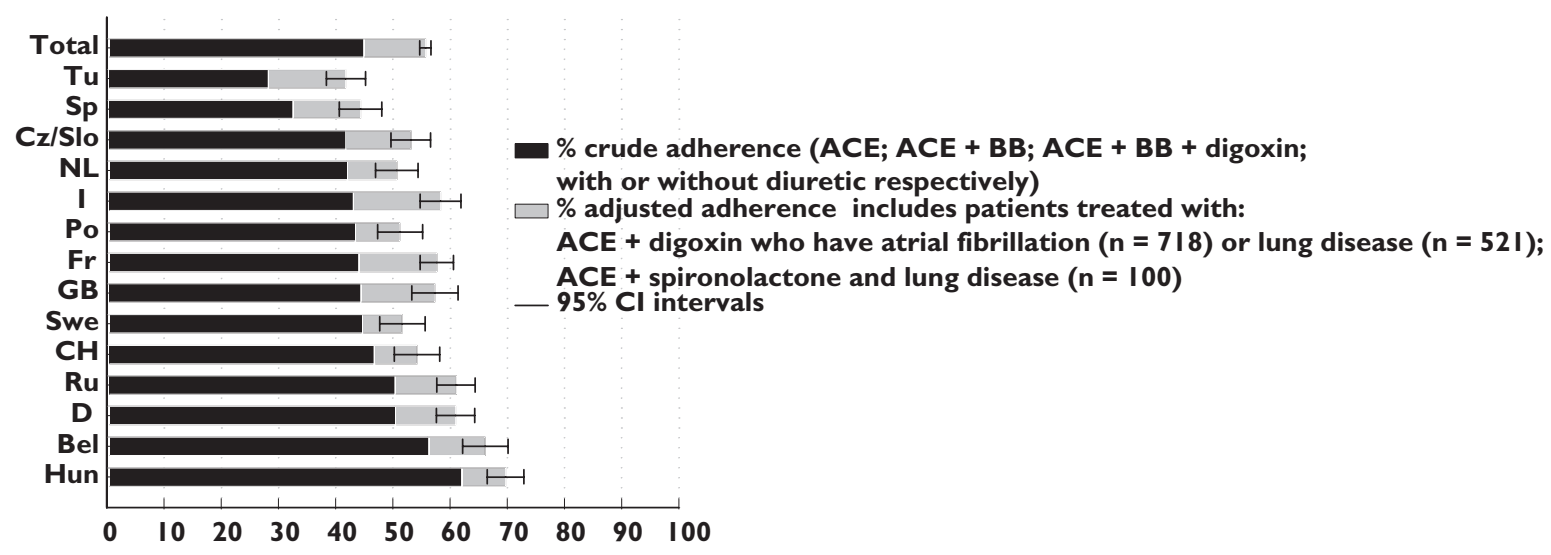

Figure 1

Prescribing according to recommendations and evidence 


\section{Effect of age on P-glycoprotein blood brain barrier transport function studied with ["C](R)-verapamil and PET}

\author{
J. R. Toornvliet, ${ }^{1}$ B. N. M. van Berckel, ${ }^{2}$ G. Luurtsema, ${ }^{2}$ M. \\ Lubberink, ${ }^{2}$ A. A. Geldof, ${ }^{2,3}$ T. M. Bosch, ${ }^{4}$ A. A. Lammertsma ${ }^{2}$ \& \\ E. J. F. Franssen ${ }^{5}$ \\ 'Dept. Pharmacy, ${ }^{2}$ Dept. Nuclear Medicine \& PET Research, \\ ${ }^{3}$ Dept. Urology, VU University Medical Centre, Amsterdam \\ ${ }^{4}$ Dept. Pharmacy, Slotervaart Ziekenhuis, Amsterdam ${ }^{5}$ Dept. \\ Pharmacy, Onze Lieve Vrouwe Gasthuis, Amsterdam
}

Introduction: $\mathrm{P}$-glycoprotein $(\mathrm{P}-\mathrm{gp})$ is an efflux-transporter, which is a major factor in the transport of various drugs across the blood brain barrier (BBB). It has been postulated that loss of P-gp function with age may be one factor in the development and progression of neurodegenerative diseases. Verapamil is a substrate for P-gp, located in the BBB. At equilibrium, the brain/blood partition coefficient of $\left[{ }^{11} \mathrm{C}\right](R)$-verapamil (inversely) reflects $\mathrm{P}$-gp function in the $\mathrm{BBB}^{1}$.

Aim: The purpose of the present study was to asses the effects of ageing on BBB P-gp function. Furthermore, the relationship with Pgp activity in white blood cells was investigated.

Methods: Five young (age 21-27 years) and 5 elderly (age 59-68 years) healthy volunteers were prospectively included in this study. All volunteers underwent two identical PET procedures with tracer dosages of $\left[{ }^{11} \mathrm{C}\right](R)$-verapamil. The brain/blood partition coefficient was calculated using Logan analysis ${ }^{2}$.

P-gp activity in white blood cells was measured using flow cytometry by measuring efflux of fluorescent substrates with or without Pgp inhibitor. P-gp activity was detected as median fluorescence intensity (MFI). Higher MFI reflects higher P-gp activity.

\section{Table 1}

brain/blood partition coefficient (pc) of $\left[{ }^{11} \mathrm{C}\right](R)$ -

verapamil and P-gp activity (MFI) for the two age groups

\begin{tabular}{llllll}
\hline Age & pc & $\begin{array}{l}\text { Activity } \\
\text { (MFI) }\end{array}$ & Age & pc & $\begin{array}{l}\text { Activity } \\
\text { (MFI) }\end{array}$ \\
\hline 21 & 0.72 & 2.88 & 59 & 0.71 & 1.43 \\
24 & 0.53 & 4.21 & 60 & 0.85 & 1.53 \\
25 & 0.60 & 2.34 & 61 & 0.75 & 2.64 \\
26 & 0.56 & 2.48 & 63 & 0.64 & 1.47 \\
27 & 0.63 & 2.53 & 68 & 0.79 & N.D. \\
\hline
\end{tabular}

Results: Results are shown in table 1. Average $( \pm \mathrm{SD})$ brain/blood partition coefficients for young and elderly volunteers was 0.61 $( \pm 0.06)$ and $0.75( \pm 0.07)$, respectively. This difference was significant $(\mathrm{p}<0.05)$. Average MFI values for elderly and young volunteers were $1.76( \pm 0.58)$ and $2.88( \pm 0.77)$, respectively. Both results indicate decreased P-gp activity in elderly volunteers.

Conclusion: This prospective study showed significantly decreased P-gp activity, i.e. reduced integrity of the BBB transport function, during ageing. Consequently, the brain may be exposed to higher drug and toxin levels in elderly subjects.

1 Bart J, et. al. Neuroimage. 2003 Nov; 20(3): 1775-82.

2 Logan et. al. J Cereb Blood Flow Metab 1990; 10: 740-7.

\section{Characteristics and predicting factors of adherence in patients with rheumatoid arthritis}

B. van den Bemt, ${ }^{1}$ W. van Lankveld, ${ }^{2}$ F. van den Hoogen, ${ }^{2,3}$

P. van Riel, ${ }^{3}$ Y. Hekster ${ }^{4}$ \& B. Benraad'

Departments of Pharmacy and Rheumatology', St Maartens

Hospital ${ }^{2}$, Nijmegen, Departments of Rheumatology ${ }^{3}$ and

Clinical Pharmacy ${ }^{4}$, UMCN St Radboud, Nijmegen, The

Netherlands

Introduction: Prescribing drugs does not ensure optimal intake. However, the patient's exposure to drugs is the crucial determinant of the effectiveness and safety of a drug. An important factor influencing drug intake is patient adherence. A number of reviews shows that adherence among patients with chronic diseases averages $50 \%$, which has medical and economic consequences. Therefore, this study was conducted to investigate drug use in patients with rheumatoid arthritis (RA), as well as co-medication and adherence to drug therapy. The aim of the study is to describe socio-economic, as well as health system-, patient- and therapy related variables associated with non-adherence.

Methods: 200 consenting outpatients using DMARDS are invited for a standardised interview. For each drug, the patient was asked about the way of drug intake, perceived effect, side-effects and adherence. The verbal answers were noted. After this interview the patients received a set of standardised self-report questionnaires assessing 1)

\section{Table 1}

Co-medication of the interviewed patients $(n=100)$

$\%$ of patients taking ...

NSAID $68 \%$

Folic acid

Drugs affecting the GI-tract

Paracetamol

Cardiovascular drugs

Supplements/vitamins/homeopathy (folic acid excluded)

Drugs against osteoporosis

Other drugs

adherence, 2) coping, 3) beliefs about medicines, 4) satisfaction about medicine information, and finally 5) overall health.

Results: Analysis of the first 100 cases showed an average drug use of 5.8 drugs/day (2-19 drugs/day). The co-medication of patients with RA comprised mainly NSAID's, peptic drugs and cardiovascular drugs (table 1). Adverse effects were reported by $49 \%$ of the patients. A total of 119 adverse effects were reported, mainly affecting the GI-tract and the central nervous system. In the interviews 
more then $95 \%$ of the patients reported that they skip medication less then once a month. This was in contrast to the scores of the selfreport Compliance Questionnaire Rheumatology (CQR) [1]. The results of the CQR suggesed that only $63 \%$ of the patients are compliant with prescribed drugs (figure 1).

Conclusion: Patients with RA use several drugs simultaneously, and a large proportion of patients has to deal with side-effects of medication. There is a discrepancy between compliance assessed verbally or by standardised questionnaire. The results of the CQR indicate that only $63 \%$ of the patients are adherent. Further analysis of our data will identify variables affecting adherence enabling us to develop individualised interventions to improve drug therapy.

1 de Klerk E, van der Heijde, Landewe R, van der Tempel, van der Linden. The compliance-questionnaire-rheumatology compared with electronic medication event monitoring: a validation study. J Rheumatol 2003; 30: 2469-75.



Figure 1

\section{Anti-Inflammatory effects of rosiglitazone in type 2 diabetes mellitus patients}

M. B. A. van Doorn, ${ }^{1}$ M. Kemme, ${ }^{1}$ M. Ouwens, ${ }^{2}$ E. J. van Hoogdalem, ${ }^{3}$ X. Liu, ${ }^{4}$ Q. S. Li, ${ }^{5}$ M. L. de Kam, ${ }^{1}$ J. Burggraaf ${ }^{1}$ \& A. F. Cohen

'Centre for Human Drug Research, Leiden, The Netherlands; 'Leiden University; Department of Molecular Biology, Leiden, The Netherlands; ${ }^{3}$ J JPRD, Beerse, Belgium; ${ }^{4}$ J JPRD, La Jolla, CA, USA; ${ }^{5}$ \&JPRD, Raritan, NJ, USA

Introduction: There is increasing recognition that chronic inflammation plays a role in the pathogenesis of insulin resistance, type 2 diabetes mellitus (T2DM) and atherosclerosis. Markers of subclinical inflammation, in particular C-reactive protein (CRP) and interleukin6 (IL-6), have shown to be powerful independent predictors of T2DM and cardiovascular disease (CVD) risk. Pre-clinical studies as well as recent clinical studies have shown that peroxisome proliferator-activated receptor- $\gamma($ PPAR- $\gamma$ ) agonists may exert their antiinflammatory actions by controlling inflammatory pathways through transcriptional mechanisms. These anti-inflammatory effects may be an integral part of the insulin sensitizing mechanism and hold the promise of reduced CVD. To follow up these observations, we investigated the baseline differences as well as the effects of rosiglitazone (RSG) on several plasma markers of inflammation (CRP, IL-6, IL$1 \beta$, Tumor necrosis factor $\alpha(\mathrm{TNF} \alpha)$ and white blood cell count (WBC)) in T2DM patients and healthy volunteers (HV) as part of a T2DM biomarker discovery trial.

Methods: 16 T2DM and $16 \mathrm{HV}$ subjects were included in this randomized, double-blind, placebo-controlled trial.
All T2DM patients stopped taking their regular oral antidiabetic medication for the duration of the study.

All subjects received RSG $4 \mathrm{mg}$ b.i.d. for 6 weeks following a 2 week placebo run-in period. An oral glucose tolerance test (OGTT) was performed before and after 6 weeks of treatment. Fasting concentrations of inflammation markers (CRP, IL-6, IL1 $\beta$, TNF $\alpha$ and WBC) and glycaemic control parameters (glucose, insulin, Cpeptide, fructosamine and $\mathrm{HbA1c}$ ) were measured at 0,2, 4, 5 and 6 weeks. Statistical analysis was performed using a repeated measures mixed effects model.

Results: Baseline CRP and IL-6 concentrations were significantly higher (184\% CI 28.2-529.8 and 96\% CI 32.8-188.4, respectively) in the T2DM group versus healthy subjects, whereas differences in IL-1 $\beta$, TNF $\alpha$ levels and WBC were not statistically significant. After RSG treatment there was a sigificant decrease from baseline in glucose (16\% CI 4.8-26.1), C-peptide (20\% CI 4.9-31.8), Insulin $(28 \% \mathrm{p}=0.008)$ and Fructosamine $(13 \%$ CI 20.7-3.7) paralled by a decrease in IL-6 (32\% 9.1-48.9), CRP (44\% - 1.96-9.2) and leukocyte count $(13 \% \quad 0.8-23.7)$ in the T2DM group. There was no significant change from baseline in IL- $1 \beta$ and TNF $\alpha$ concentrations. In the HV group there were no significant treatment effects apart from a decrease in peak insulin and glucose concentrations after OGTT (26\% 1.9-43.5 and 16\% 0.4-29.7, respectively).

Conclusions: The results of this study support the concept that inflammation is a feature of T2DM. RSG decreased IL-6, CRP and WBC paralleled by improved glycaemic control corroborating the previously observed association between anti-inflammatory effects and insulin sentisization of PPAR $\gamma$ agonists. In addition, these results indicate potentially beneficial effects on CVD risk in subjects treated with RSG. 


\section{Cyclosporine interacts with mycophenolate mofetil by inhibiting the multidrug resistance-associated protein 2}

R. M. van Hest, ${ }^{1}$ D. A. Hesselink, ${ }^{2}$ R. A. A. Mathot, ${ }^{1}$ F. Bonthuis, ${ }^{3}$ W. Weimar, ${ }^{2}$ R. W. F. de Bruin ${ }^{3}$ \& T. van Gelder, ${ }^{1,2}$

Departments of 'Hospital Pharmacy, Clinical Pharmacology

Unit, ${ }^{2}$ Internal Medicine, Renal Transplant Unit, ${ }^{3}$ Experimental

Surgery, Erasmus Medical Center, Rotterdam, The Netherlands

Background: In a previous animal study we demonstrated that cyclosporine (CsA) decreases exposure to mycophenolic acid (MPA) and increases exposure to its metabolite MPA-glucuronide (MPAG), possibly by interfering with the biliary excretion of the latter. In contrast, the calcineurin inhibitor (CNI) tacrolimus (Tac) does not influence the pharmacokinetics of MPA and MPAG. We aimed to elucidate the role of the multidrug resistance-associated protein (MRP)-2 in the interaction between mycophenolate mofetil (MMF) and CsA.

Methods: Three groups of ten Mrp2-deficient rats (TR- rat) were treated for 1 week with either vehicle, CsA $(8 \mathrm{mg} / \mathrm{kg})$ or Tac $(4 \mathrm{mg} / \mathrm{kg}$ ) by oral gavage. Hereafter, co-administration with MMF $(20 \mathrm{mg} / \mathrm{kg}$ ) was started in all groups and continued through day 14. The $24 \mathrm{~h}$ MPA/MPAG area-under the concentration-time curve
(AUC) was determined after single (day 7) and multiple MMF doses (day 14). MPA and MPAG concentrations were determined using HPLC.

Results: On day 7 and 14, there were no differences in the mean $( \pm \mathrm{SD})$ MPA AUC between the CsA and Tac-treated rats: $25 \pm 6$ vs $22 \pm 6$ and $30 \pm 13$ vs $28 \pm 10 \mathrm{mg} \times \mathrm{h} / \mathrm{L}$ (for days 7 and 14 , respectively; $\mathrm{P}=1.0$ ). Also, MPAG exposure was not different between CsA and Tac-treated animals on both study days: $377 \pm 87$ vs $422 \pm$ 54 and $365 \pm 75$ vs $366 \pm 58 \mathrm{mg} \times \mathrm{h} / \mathrm{L}(\mathrm{P}=0.54$ and $\mathrm{P}=0.28)$. Interestingly, MPA exposure in the vehicle group on day 7 (32 \pm $8 \mathrm{mg} \times \mathrm{h} / \mathrm{L})$ and on day $14(42 \pm 6 \mathrm{mg} \times \mathrm{h} / \mathrm{L})$ was significantly higher than in the Tac group $(\mathrm{P}<0.01$ and $\mathrm{P}=0.03$, respectively). The MPA exposure in the vehicle group was also higher compared with CsA-treated animals but this difference was not statistically significant $(\mathrm{P}=0.07$ and $\mathrm{P}=0.06$ for days 7 and 14 , respectively).

Conclusion: In contrast to normal rats, the pharmacokinetics of MMF are not different in Mrp2-deficient rats receiving either CsA or Tac as co-medication. This finding shows that inhibition of Mrp2 by CsA contributes to the interaction between CsA and MMF. However, in the Mrp2-deficient rat, CNIs do influence MMF pharmacokinetics as compared with placebo. This observation may be explained by CNI-dependent blockade of alternative MPAGexcretion mechanisms operational in the Mrp2-deficient rat.

\section{The influence of ethnicity on anti-hypertensive drug effects (a Dutch study with 3 ethnic groups) \\ P. C. van Rijn-Bikker, ${ }^{1}$ G. Mairuhu, ${ }^{1}$ G. A. van Montfrans, ${ }^{1}$ H. J. Guchelaar $^{2}$ \& R. P. Koopmans ${ }^{1}$ \\ 'Academic Medical Centre Amsterdam, The Netherlands; 'Leiden University Medical Centre, Leiden, The Netherlands}

Aims: The ROTATIE trial is a cross-over open label treatment study aimed at deriving a predictive model of individual anti-hypertensive drug effects. To determine if ethnicity is a predictor of these effects we have assessed the influence of ethnicity on the lowering of systolic blood pressure (SBP) and diastolic blood pressure (DBP) by all 5 primary groups of antihypertensive agents.

Methods: Participants went through five successive 6-week periods of single drug treatment (drugs see table; HCT is hydrochlorothiazide) in a randomized order, separated by 3-weeks wash-out. BP was measured during 15 minutes at rest in the seated position using an automatic device. One hundred and two patients aged 47,5 (35-60) years were included. Ethnic background was assessed before inclusion. Data of subjects that did not complete the 6-week treatment period on medication, or who did not take their medication daily, were excluded. The results were analyzed with SPSSANOVA, using SBP and DBP lowering as dependent variables and ethnicity and drug as fixed factors. Interactions between the fixed factors were included in the model. Results are expressed as means (in $\mathrm{mm} \mathrm{Hg}$ ) with $95 \%$ confidence interval.

Results: In total we analyzed 379 treatment periods (144 of Caucasians, 175 of Blacks, 60 of Hindustani). There were considerable variations in drug effects between the ethnicgroups for both SBP and DBP lowering ( $\mathrm{p}=0.01$ and $\mathrm{p}=0.05$, respectively; tables 1,2$)$. In the ANOVA-analysis the factors ethnicity and drug were not significant for SBP $(p=0.18$ and $p=0.45)$. For DBP there was no difference between the drugs $(\mathrm{p}=0.08)$, however Blacks and Hindustani had more effect than Caucasians ( $\mathrm{p}=0.048)$. Probably this is due to a higher DBP at inclusion of the latter two groups.

Conclusion: Ethnicity is a strong predictor of anti-hypertensive drug effects. The third generation $\beta$-blocker was the only drug that was effective in all 3 ethnic groups. The efficacy of the 5 drugs on SBP and DBP shows almost the same ethnic pattern.
Table 1

SBP-lowering (italics means statistical significance)

\begin{tabular}{lllr}
\hline SBP-lowering & Caucasians & Blacks & Hindustani \\
\hline Eprosartan & $5.7(1.6 ; 9.6)$ & $2.3(-1.5 ; 6.2)$ & $6.1(-0.1 ; 12.3)$ \\
Nebivolol & $7.4(3.4 ; 11.4)$ & $5.3(1.7 ; 9.0)$ & $10.1(3.7 ; 16.6)$ \\
Barnidipine & $1.3(-2.9 ; 5.5)$ & $9.8(5.7 ; 13.8)$ & $8.5(1.4 ; 15.6)$ \\
Lisinopril & $8.6(4.2 ; 12.9)$ & $4.2(0,4 ; 7.9)$ & $12.2(5.8 ; 18.7)$ \\
HCT & $3.4(-0.9 ; 7.7)$ & $10.3(6.7 ; 14)$ & $5.7(-0.5 ; 11.9)$ \\
\hline
\end{tabular}




\begin{tabular}{lllll}
\cline { 1 - 2 } DBP-lowering & Caucasians & Blacks & Hindustani & $\begin{array}{l}\text { Table 2 } \\
\text { DBP-lowering (italics means statistical } \\
\text { significance) }\end{array}$ \\
\cline { 1 - 3 } Eprosartan & $3.5(0.3 ; 6.6)$ & $2.6(-0.4 ; 5.6)$ & $3.4(-1.4 ; 8.3)$ \\
Nebivolol & $5.2(2.1 ; 8.4)$ & $7.7(4.9 ; 10.6)$ & $9.2(4.2 ; 14.2)$ \\
Barnidipine & $1.9(-1.4 ; 5.2)$ & $8.5(5.3 ; 11.6)$ & $6.6(1.0 ; 12.1)$ \\
Lisinopril & $4.9(1.6 ; 8.3)$ & $2.5(-0.5 ; 5.4)$ & $9.6(4.6 ; 14.7)$ \\
HCT & $2.4(-1.0 ; 5.7)$ & $7.0(4.2 ; 9.9)$ & $3.1(-1.7 ; 7.9)$ \\
\hline
\end{tabular}

\section{Duration of response on tamoxifen in advanced breast cancer depends on CYP2C19 genotype}

R. H. N. van Schaik, E. Teuling, I. van der Heiden, M. van Fessem, I. van Staveren, M. van Vliet, M. Look, J. Klijn, J Foekens, J. Lindemans \& E. Berns

Depts Clinical Chemistry and Medical Oncology, Erasmus MC Rotterdam, The Netherlands

Metastatic breast cancer is often treated with tamoxifen. Resistance to anti-estrogens, however, is a major problem in breast cancer treatment. Tamoxifen is metabolized by cytochrome P450 (CYP) enzymes: activation to the 50 -fold more potent $4 \mathrm{OH}$ tamoxifen is mainly catalyzed by CYP2D6 while inactivation to N-desmethyltamoxifen depends on CYP3A4 and CYP3A5 activity. Also CYP2B6, CYP2C9 and CYP2C19 have been described to play a role in tamoxifen metabolism. Genetic polymorphisms in CYPs, affecting enzymatic activity, may influence the outcome of tamoxifen therapy. We hypothesize that CYP2D6 poor metabolizers (approximately 7\% of the general population) would be overrepresented in the tamoxifen non-responder group.
We developed and validated genotyping analyses for the variant alleles CYP2D6*3, *4, *5, *6, CYP2B6*5, CYP2C9*2, *3 and CYP3A5*3. Genomic DNA of 282 retrospectively collected primary breast tumor samples of patients with advanced disease were analysed for the variant alleles. Results were compared to patient and tumor characteristics. Of the investigated patients, 55 showed response, 113 had stable disease $>6$ months, 21 had stable disease $<6$ months and 93 had progressive disease. None of the patients received adjuvant therapy.

CYP2D6 poor metaboliser status $(n=21)$ did not correlate with tamoxifen response. Also CYP2C9, CYP3A5 and CYP2B6 genotype did not show any correlation with response. However, CYP2C19*1/*1 genotype $(\mathrm{n}=196)$ was significantly associated with a shorter time to tumor progression (hazard ratio 1.5; $95 \%$ C.I. $1.2-2.1 ; \mathrm{p}=0.004)$.

We showed that duration of response to tamoxifen in our study correlates with CYP2C19 genotype. Further studies are needed to investigate whether inhibition of CYP2C19 activity may indeed prolong the response to tamoxifen.

\section{Imipramine dose requirement in depressed patients is predicted by CYP2D6 functional gene dosage}

R. H. N. van Schaik, M. van Fessem, M. van Vliet, J. Verploeg,

A. G. Vulto, R. A. A. Mathot, J. A. Bruijn, J. Lindemans,

T. van Gelder \& P. W. Schenk

Depts Clinical Chemistry, Hospital Pharmacy and Psychiatry,

Erasmus MC Rotterdam, The Netherlands

The tricyclic antidepressant imipramine has a narrow therapeutic window, yet a 50-fold variation in plasma levels is observed when administered at a standard dose. It is metabolised by cytochrome P450 (CYP) enzymes, in which it is converted to the active metabolite desipramine by CYP2C19. In turn, desipramine is inactivated by CYP2D6 to 2OH-desipramine. Therapy for depressed patients consists of adjusting imipramine dose to obtain combined plasma levels for imipramine + desipramine within a predefined window. For both CYP2C19 and CYP2D6, variant alleles encoding inactive enzymes exist, resulting in poor metabolisers deficient in CYP2C19 (2\% of Caucasians) or CYP2D6 (7\% of Caucasians) activity. The presence of multiple alleles can result in ultra rapid drug metabolism. Correlations between genotype and plasma levels have been described in small groups. In this study, we investigated the impact of gene dosage (i.e. the number of functional alleles in which null-alleles and decreased activity alleles are taken into account) on imipramine dose requirement in patients suffering from depression.

In 178 depressed, weight, steady-state imipramine dose and plasma levels of both imipramine and desipramine were recorded. From EDTA blood, genomic DNA was isolated and analysed for the CYP2D6 null alleles $* 3, * 4, * 5, * 6$ and the decreased activity alleles $* 9, * 10, * 41$. In addition, the presence of the CYP2D6 gene duplication was investigated. Based on the genotyping results, functional gene dosages $(0$ to $>2)$ were assigned. In addition, genotyping for the CYP2C19 alleles was performed.

We found a significant correlation between steady-state imipramine dose and CYP2D6 genotype: gene dosage groups $0,0.5$, $1,1.5,2$ and $>2$ had imipramine dosages of $118 \pm 14,154 \pm 18,190$ $\pm 10,198 \pm 14,278 \pm 16$ and $309 \pm 32 \mathrm{mg}$ (mean \pm s.e.m.), respectively (ANOVA, p < 0.0001). Dose-corrected plasma levels of imipramine + desipramine, or desipramine alone, also correlated with CYP2D6 functional gene dosage (ANOVA, $\mathrm{p}<0.0001$ ).

We conclude that imipramine exposure is highly correlated to the number of functional CYP2D6 alleles. Determining CYP2D6 gene dosage prior to initiation of imipramine therapy may aid in achieving the optimal imipramine dose more quickly. 


\section{Elucidating the mechanism of lithium acquired nephrogenic diabetes insipidus in patients under chronic lithium treatment}

I. Wilting, ${ }^{1,2}$ R. Baumgarten, ${ }^{3}$ K. L. L. Movig, ${ }^{4}$ J. van Laarhoven, ${ }^{5}$

A. J. Apperloo, ${ }^{6}$ W. A. Nolen, ${ }^{7}$ E. R. Heerdink' \& A. C. G. Egberts ${ }^{1,2}$

'Department of Pharmacoepidemiology and Pharmacotherapy,

Utrecht Institute for Pharmaceutical Sciences, Utrecht University,

Utrecht, ${ }^{2}$ Department of clinical pharmacy, TweeSteden hospital

and St Elisabeth Hospital, Tilburg, ${ }^{3}$ Department of clinical

chemistry, Atrium Medical Center, Heerlen, ${ }^{4}$ Department of

Clinical Pharmacy, Medisch Spectrum Twente, Enschede,

${ }^{5}$ Department of psychiatry, St Elisabeth Hospital, Tilburg,

${ }^{6}$ Department of nephrology, St Elisabeth Hospital, Tilburg,

'Department of psychiatry, University of Groningen Medical

Centre, Groningen, The Netherlands

Introduction: Acquired nephrogenic diabetes insipidus (NDI) is a well-known complication of lithium treatment (Boton et al. 1987). NDI is defined as the inability of the kidney to concentrate urine in response to vasopressin, resulting in the production of large quantities of hypotonic urine. In normal physiology vasopressin (AVP) is released in response to either an increase in serum osmolality or a strong decrease in circulating volume. AVP is subsequently bound to vasopressin-type-2 receptors (V2R) located on the basolateral side of the distal tubular cells, followed by both an elevation of cAMP and apical insertion of aquaporin-2 (AQP-2) (water channels). This leads to an increased water reabsorption from pre-urine and thereby to more concentrated urine. The objective of our study was; to elucidate which part of the V2R-cAMP-AQP-2 cascade is involved in the development of lithium induced NDI.

Methods: We conducted a study in 20 patients under chronic lithium treatment, either with $(n=10)$ (24-hour urine volume $\geq 3 \mathrm{~L}$ ) or without polyuria $(\mathrm{n}=10)$. We determined the kidney urine concentrating activity in a situation of minimal stimulation (induced by water loading), followed by maximal stimulation (induced by nasal administration of 1-desamino-8-D-arginine-vasopressine (dDAVP)). We monitored both cAMP and AQP-2 excretion and urine osmolality. Urine was collected every hour, starting 2 hours before until 4 hours after dDAVP administration.

Results: The partial correlation $\left(\mathrm{R}^{2}\right)$ between cAMP excretion and urine osmolality was 0.94 . For all patients individually, a significant correlation $(\mathrm{p}<0.05)$ between urine cAMP excretion and urine osmolality was established. No significant partial correlation was observed between urine AQP-2 excretion and urine osmolality nor between urine cAMP and AQP-2 excretion.

Conclusion: We found that in lithium induced NDI in man, the cAMP generation in response to dDAVP administration, after water loading, is impaired. It remains to be elucidated whether G-proteins or adenylate cyclase are the major targets for the mechanism underlying lithium induced NDI in man.

Boton R, Gaviria M, Batlle D. Prevalence, pathogenesis, and treatment of renal dysfunction associated with chronic lithium therapy. American Journal of Kidney Diseases 1987; 10(5): 329-45. 